\section{We've got mail!}

With January comes the usual lot of New Year's resolutions. One of Nature Methods' resolutions is to foster a dialog with its readers. For this purpose, a new Correspondence section makes its January debut in the following pages to receive your letters. One of the motivations is to encourage a two-way flow of information and give you the opportunity to comment on articles published in the journal. But also, we believe and hope that, with the journal's focus on practical issues, the Correspondence section can develop into a forum for readers to exchange a wide range of useful information.

As in traditional Correspondence sections, we expect your letters to be triggered by the reading of articles and opinion pieces in the journal. A quick survey of similar letters in other scientific publications has set our expectations for a substantial amount of criticism and dissent. We anticipate that our readers will want to highlight limitations of certain methodologies - not only those that may occasionally have escaped the prepublication peer review, but also those that simply can only be revealed after the technology has been extensively used and expanded to various applications - and we recognize the value of these discussions for the scientific community.

However, brimming with January optimism and good resolutions, we dare to think that letters with a positive spin will also find their ways to our desks. The journal's focus on methods and tools for research should indeed be the drive for this positive expansion. For example, positive followups on published articles could complement the methods with additional tips or variations likely to expand their scope. In addition, a crucial point in publication of methods, and especially protocols, is their need for updates. Technology is moving fast, and without a system to track improvements, a specific methodology described today in an article or a protocol may very well become obsolete as soon as a year from now. We strongly encourage the original authors and further developers of a technique to write in order to keep readers apprised of significant developments. If published as a Correspondence, these additions will benefit online from bidirectional linking to and from the original paper, allowing the records to be updated as well.
We also hope that the scope of this section will not be limited to subjects that have been explicitly treated in our pages. In the scientific community, there are a lot of cautionary tales making their way by word of mouth from one laboratory to another or being discussed in the hallways of conference centers. These stories have to do with the design of experiments, use of controls or interpretation of data from a particular assay. They are not criticizing a particular article or laboratory; they usually are the result of personal observations and individual frustration experienced in the struggle with the assay in question. In some cases, dissemination of these cautionary tales may be extremely beneficial to the community, to avoid mistakes or unintentional misuses. Publication of a Correspondence piece, having passed the peer review test, will be more far-reaching than hallway whispers.

Furthermore, we would like the Correspondence section to become a forum for scientists to communicate information and tools other than methods that may benefit the research of many. The letter inaugurating the new section this month is, in our opinion, a good example. It comes from a group of academic researchers who have engaged in the construction of a repository of data relative to protein purification and, specifically, technical information on refolding proteins produced in bacteria. The effort to build this 'knowledge base' is considerable, but the amount of data in the library, although already substantial, has been limited to published data and occasional contributions. In addition to positive comments by peer reviewers on the quality and functionality of the tool, we received assurance from the authors that they have the resources to sustain this effort to curate and expand the database into an increasingly useful tool for the structural genomics community as well as for individual researchers struggling with the refolding of their favorite protein. Therefore, along with the developers of this tool, we hope that wide dissemination of the news of their enterprise will encourage people to contribute their unpublished data and enhance the usefulness of the database.

Nature Methods' Correspondence is thus a flexible format, and we want to adapt it to the needs of the community. So, pick up your pens, we want to hear from you! 